\title{
Heavy Metal, Organochlorine Pesticide and Polychlorinated Biphenyl Contamination in Arctic Ground Squirrels (Spermophilus parryi) in Northern Alaska
}

\author{
S.M. ALLEN-GIL, ${ }^{1}$ D.H. LANDERS,${ }^{2}$ T.L. WADE,${ }^{3}$ J.L. SERICANO,${ }^{3}$ B.K. LASORSA,${ }^{4}$ \\ E.A. CRECELIUS ${ }^{4}$ and L.R. CURTIS ${ }^{5}$
}

(Received 5 February 1997; accepted in revised form 11 August 1997)

\begin{abstract}
Heavy metal and organochlorine (OC) concentrations, including organochlorine pesticides and polychlorinated biphenyl congeners (PCBs), were determined in arctic ground squirrels (Spermophilus parryi) from three sites in the Brooks Range of northern Alaska in 1991-93. Heavy metals were present in most squirrel livers collected, with concentrations of trace elements (As, $\mathrm{Cd}, \mathrm{Hg}, \mathrm{Ni}$, and $\mathrm{Pb}$ ) averaging below $1 \mu \mathrm{g} / \mathrm{g}$ wet weight. Hexachlorobenzene (HCB), p,p'-DDE, gamma hexachlorocyclohexane $(\gamma-\mathrm{HCH})$, trans-nonachlor, and PCBs 138,153 , and 170 were the most frequently detected OCs in fat and liver. Average concentrations of individual $\mathrm{OC}$ analytes were below $20 \mathrm{ng} / \mathrm{g}$ wet weight in liver and below $15 \mathrm{ng} / \mathrm{g}$ wet weight in fat. Rank correlations indicate that concentrations of heavy metals and of OCs accumulate in concert with one another (As, Cd, $\mathrm{Cu}$, and $\mathrm{Zn} ; \mathrm{PCBs} 138,170$, and 180). Although heavy metal and OC concentrations are low relative to other areas and other arctic species, the occurrence of these compounds illustrates the global pervasiveness of persistent organic compounds and the potential for bioaccumulation in the terrestrial arctic food web.
\end{abstract}

Key words: heavy metals, organochlorine pesticides, polychlorinated biphenyls, Spermophilus parryi, northern Alaska

RÉSUMÉ. De 1991 à 1993, on a mesuré les concentrations en métaux lourds et en organochlorés, y compris des pesticides organochlorés et des congénères du diphényle polychloré (PCB), chez le spermophile arctique (Spermophilus parryi) à trois endroits situés dans la chaîne de Brooks de l'Alaska septentrional. On a trouvé des métaux lourds dans la plupart des foies de spermophiles prélevés, avec des concentrations en éléments traces ( $\mathrm{As}, \mathrm{Cd}, \mathrm{Hg}, \mathrm{Ni}$ et $\mathrm{Pb}$ ) inférieures en moyenne à $1 \mu \mathrm{g} / \mathrm{g}$ de poids frais. L'hexachlorobenzène (HCB), le p,p'-DDE, l'hexachlorocyclohexane gamma (HCH- $\gamma$ ), le trans-nonachlore et les PCB 138, 153 et 170 étaient les organochlorés les plus fréquemment détectés dans le tissu adipeux et le foie. Les concentrations moyennes des organochlorés analysés individuellement étaient inférieures à $20 \mathrm{ng} / \mathrm{g}$ de poids frais pour le foie et à $15 \mathrm{ng} / \mathrm{g}$ de poids frais pour le tissu adipeux. Les corrélations de rang révèlent que les concentrations en métaux lourds augmentent de concert avec celles en organochlorés (As, Cd, Cu et Zn; PCB 138, 170 et 180). Bien que les concentrations en métaux lourds et en organochlorés soient faibles par rapport à celles d'autres régions et à celles d'autres espèces arctiques, la présence de ces composés illustre l'omniprésence planétaire de composés organiques persistants et le potentiel pour une accumulation biologique dans le réseau trophique terrestre de l'Arctique.

Mots clés: métaux lourds, pesticides organochlorés, diphényles polychlorés, Spermophilus parryi, Alaska septentrional

Traduit pour la revue Arctic par Nésida Loyer.

\section{INTRODUCTION}

Arctic terrestrial food webs are characterized by low diversity and strong interactions within communities. The low diversity reflects the fact that few species are adapted to the harsh environment; only 50 of the world's 4000 mammals are found in the Arctic, and only 8 bird species are year-round residents (Stonehouse, 1989). The faunal base of the terrestrial food web is centered on a small number of rodent species. Given the low diversity of rodent prey species, most arctic predators are opportunists, exhibiting considerable overlap in their diets (Hoffmann, 1974). Annual fluctuations in levels, longer term cycling in populations, and the coupling between prey and predator population cycles are more

\footnotetext{
${ }^{1}$ Department of Fisheries and Wildlife, 104 Nash Hall, Oregon State University, Corvallis, Oregon 97331, U.S.A.; present address: National Research Council Associate, U.S. EPA National Health and Environmental Effects Research Laboratory, 200 SW 35th St., Corvallis, Oregon 97333, U.S.A.; allens@ mail.cor.epa.gov

${ }^{2}$ U.S. EPA National Health and Environmental Effects Research Laboratory, 200 SW 35th St., Corvallis, Oregon 97333, U.S.A.

${ }^{3}$ Geochemical and Environmental Research Group, Texas A\&M University, 833 Graham Road, College Station, Texas 77845, U.S.A.

${ }^{4}$ Battelle Pacific Northwest Division, 1529 West Sequim Bay Road, Sequim, Washington 98382, U.S.A.

${ }^{5}$ Department of Fisheries and Wildlife, 104 Nash Hall, Oregon State University, Corvallis, Oregon 97331, U.S.A.; present address: Department of Environmental Health, College of Public and Allied Health, East Tennessee State University, Johnson City, Tennessee 37614-0682, U.S.A.

(C) The Arctic Institute of North America
} 
pronounced for arctic species than for those in temperate environments (Remmert, 1980).

The cyclic nature of arctic rodent populations potentially renders them susceptible to chronic toxicological impact. It is believed that relationships among species and external stresses (severe winters, limited food availability) are critical factors in determining the magnitude and length of population level cycles (Remmert, 1980). The influence of contaminantinduced physiological stress may exacerbate rapid population declines. Since re-establishment of the population following a population decline is dependent on the reproductive fitness of relatively few individuals and the survivorship of their offspring, the effects of agents that are genotoxic, teratogenic, or otherwise detrimental to reproduction may be amplified. Such effects on rodent populations may have repercussions for predator populations relying on small mammals as prey.

A physiological adaptation of many arctic animals to the harsh winters is the deposition and utilization of fat reserves (Batzli, 1981). Ground squirrels, in particular, rely heavily on fat deposits as an energy source during their 7 to 9 months of hibernation (Behirsch, 1984; Kenagy and Barnes, 1988). This feature renders animals susceptible both to the accumulation of persistent organic compounds and to the potential for enhanced toxicity caused by mobilization of fat reserves which may be laden with lipophilic organic compounds (Geyer et al., 1990; Shore and Douben, 1994).

Transfer of lipophilic organic compounds from prey to predator is a well-established process. Biomagnification has been documented for airborne pollutants in arctic ecosystems (Andersson et al., 1988; Norstrom et al., 1988; Larsson et al., 1990b; Kidd et al., 1995). In terrestrial systems, Larsson et al. (1990a) demonstrated that an increase in atmospheric deposition of DDT, lindane and PCBs increased uptake by plants and elevated levels in herbivores and, subsequently, in predators. Villeneuve et al. (1985) reported trophic transfer of OCs from lichen to reindeer to humans in northern Sweden.

Heavy metal contamination in arctic terrestrial food webs is also a concern. Natural geologic mineral enrichment, mining practices within the Arctic, and atmospheric transport from remote sources may all contribute to heavy metal accumulation in arctic fauna (Thomas et al., 1992; Landers et al., in press). Recent and fossil shells from the eastern Canadian Arctic show a five-fold increase in lead (Bourgoin and Risk, 1987). Cadmium, in particular, often occurs in high concentrations in arctic fauna (Shaw and Gunn, 1981; SnyderConn and Lubinski, 1993; Elkin and Bethe, 1995).

The primary goal of this research was to develop an increased understanding of contaminant loadings in select arctic fauna. Herbivorous small mammals with limited home range sizes are particularly well suited for biomonitoring of atmospherically deposited contaminants (Talmage and Walton, 1991). Among small herbivores with relatively confined home ranges, the arctic ground squirrel (Spermophilus parryi) was selected as a target species because of its ubiquitous distribution, reliance on fat reserves during hibernation, importance as a prey species (Carl, 1971; Jones and Theberge, 1983; Poole and Boag, 1988) and well-studied physiology and life history (e.g., Batzli and Sobaski, 1980; Baust and Brown, 1980; Chappell, 1981; Barnes, 1989). Arctic ground squirrels live in burrow colonies along river banks and lake basin rims, with average densities ranging from 1.5 to $8 \mathrm{ha}^{-1}$ in favorable habitat (Batzli and Sobaski, 1980). Animals emerge from their 8- to 9-month hibernation in April-May, breed in May, and give birth to young (5-6 per litter) in June and early July (Carl, 1971; Kiell and Millar, 1980). Ground squirrels are opportunistic herbivores, with 40 species of herbaceous dicotyledons comprising $25 \%$ to $75 \%$ of the diet. Most foraging occurs within $50 \mathrm{~m}$ of burrow sites (Batzli and Sobaski, 1980). Feeding during the summer is intense: by late August, percent body weight as fat can exceed 50\% (Batzli and Sobaski, 1980; Kiell and Millar, 1980).

\section{METHODS}

Ground squirrels were trapped in the vicinity of Elusive, Schrader, and Feniak Lakes, located in the foothills of the Brooks Range, Alaska (Fig. 1). These sites were selected by the U.S. Environmental Protection Agency's (USEPA) Arctic Contaminants Research Program as integrated sites from which lake sediments, fish tissue, moss, and lichen samples were also analyzed for heavy metal and organochlorine contaminants (see Landers et al., 1992, 1995; Ford et al., 1995; Gubala et al., 1995; Wilson et al., 1995; Allen-Gil et al., 1997). Site selection was based on a combination of factors, including acceptable lake bathymetry for interpretation of sediment cores, occurrence of target lichen, moss, small mammal, and fish species, and geographic separation. All sites were situated in a region of high atmospheric deposition (Ford and Bedford, 1987). Although still within the foothills of the Brooks Range, Feniak Lake in northwestern Alaska is at a lower elevation than Elusive or Schrader Lakes.

\section{Sample Collection}

A combination of destructive and nondestructive sampling techniques was used. In order to provide sufficient liver mass for accurate residue analyses given the predicted low concentrations, whole livers were taken from six individuals from each site. After this quota had been achieved, nondestructive techniques were implemented as described below. All tissue samples from destructive and nondestructive procedures were treated identically following removal. Animals were trapped in Tomahawk "skunk special" live traps, located in rodent runways and other heavily utilized microhabitats (after Babcock, 1986). Traps were baited with carrots, shielded from exposure to sun, wind, and rain, and checked every 4 to 6 hours. Trapped animals were transported to a base camp for processing.

Individuals were anaesthetized immediately and kept at a moderate depth of anaesthesia with methoxyflurane 


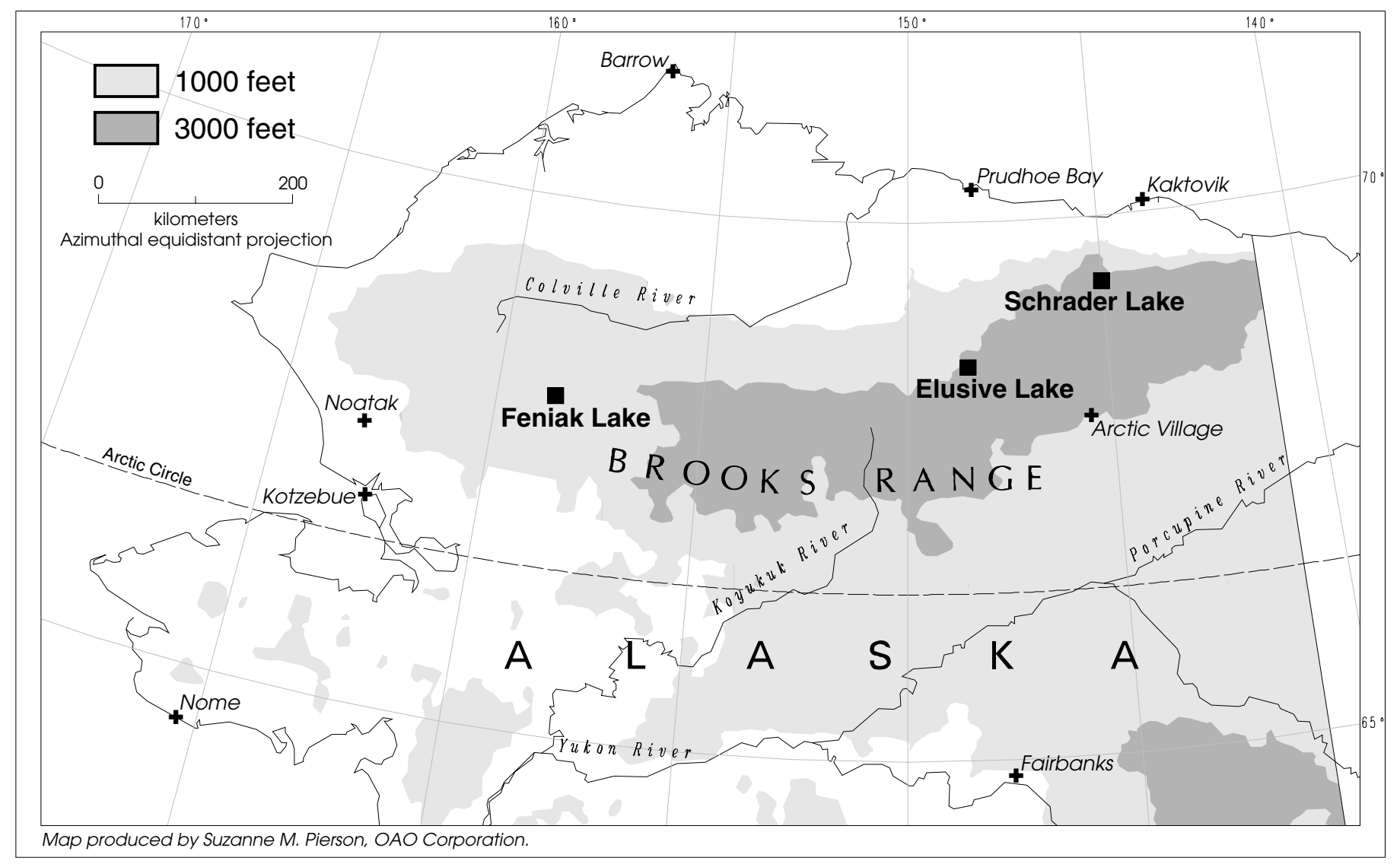

FIG. 1. Integrated sites for the USEPA's Arctic Contaminants Research Program.

$\left(\right.$ Metofane $\left.^{\mathrm{TM}}\right)$. Sex, weight, total length, and approximate age were determined on all captured animals. This information served to characterize factors that might modulate contaminant bioaccumulation. For individuals sampled destructively, liver tissue was extracted and weighed. Liver somatic index (LSI) was calculated as (liver wt/(total wt - liver wt)).

Lipid reserves were used as a parameter for evaluating intermediary fat metabolism and as an overall health indicator. Total fat content was determined using a commercial fat analyzer (EM-SCAN Model SA-2), composed of an insulated holding chamber wrapped with an electrically conductive coil. This instrument nondestructively measures the total electrical conductivity of the animal, which can be converted to lean body mass (Walsberg, 1988). The difference between the total body weight and lean body mass represents the total lipid content. The instrument was calibrated using 20 laboratory-reared ground squirrels for which whole body fat content was also determined by Soxhlet extraction following the methods of Bligh and Dyer (1959).

Nondestructive liver samples were obtained by pinch biopsy (as described by Slatter, 1985). A portion of the abdominal area was shaved and scrubbed with iodine and alcohol prior to biopsy. Fat samples were collected from the abdominal and/or gonadal region. To minimize the threat of predation, nondestructively sampled animals were placed in a trap and allowed to recover fully from the anesthesia $(\sim 12 \mathrm{hr})$ before being released at the location of capture.
Numbered metal tags were attached to individuals' ears to identify them in subsequent sampling years.

Liver samples were weighed, divided for organic and inorganic analysis, and transferred to pre-cleaned I-Chem ${ }^{\mathrm{TM}}$ jars and acid-cleaned borosilicate vials with teflon screw-tops respectively. All fat and liver (whole and biopsy) samples were frozen (or nearly frozen) on site and shipped by air to the respective laboratories (OCs: Geochemical and Environmental Research Group at Texas A\&M University; metals: Battelle Pacific Northwest).

\section{Contaminant Analysis}

Methods for heavy metal analysis have been previously reported in Allen-Gil et al. (1997). Freeze-dried samples (500 $\mathrm{mg}$ ) were analyzed for arsenic (As), cadmium (Cd), copper $(\mathrm{Cu})$, lead $(\mathrm{Pb})$, and zinc $(\mathrm{Zn})$ by inductively coupled plasma spectrometry (ICP-MS), for mercury $(\mathrm{Hg})$ by cold vapor atomic absorption (CVAA), and for selenium (Se) and nickel (Ni) by either ICP-MS or graphite furnace atomic absorption (GFAA).

Tissue OC analysis was performed by the Geochemical and Environmental Research Group at Texas A\&M University. The methods and quality assurance procedures have been reported in Allen-Gil et al. (in press). The procedures for liver and fat samples were adapted from those employed for the U.S. National Oceanic and Atmospheric Administration's Status and Trends Program and 
TABLE 1. Characteristics of arctic ground squirrels sampled from sites in northern Alaska.

\begin{tabular}{|c|c|c|c|}
\hline \multirow[b]{2}{*}{ Dates sampled } & Elusive & Schrader & Feniak \\
\hline & $\begin{array}{l}9-11 \text { August, } 1991 \\
14-21 \text { August, } 1993\end{array}$ & $\begin{array}{c}\overline{14-18 \text { August, } 1991} \\
22 \text { July }-1 \text { August, } 1992\end{array}$ & $\begin{array}{c}\text { 5-6 July, } 1992 \\
\text { 6-10 August, } 1993\end{array}$ \\
\hline $\begin{array}{l}\text { Total number sampled: } \\
\text { males/females }\end{array}$ & $\begin{array}{l}13 \\
6 / 7\end{array}$ & $\begin{array}{c}26 \\
12 / 14\end{array}$ & 13 \\
\hline Length $(\mathrm{cm})$ & $37.1 \pm 2.6^{1}$ & $38.3 \pm 2.8$ & $36.8 \pm 2.0$ \\
\hline Weight $(\mathrm{g})$ & $599 \pm 127$ & $603 \pm 141$ & $593 \pm 133$ \\
\hline Percent body weight as fat & $19.4 \pm 10.3$ & $14.4 \pm 10.8$ & $13.9 \pm 10.5$ \\
\hline Percent lipid in liver & $2.59 \pm 0.84$ & $2.24 \pm 1.31$ & $2.46 \pm 0.95$ \\
\hline Percent lipid in fat & $76.9 \pm 7.90^{\mathrm{a}}$ & $41.2 \pm 10.6^{\mathrm{b}}$ & $30.1 \pm 24.9^{\mathrm{b}}$ \\
\hline Percent dry weight in liver & $30.4 \pm 1.4$ & $38.5 \pm 12.8$ & $30.8 \pm 7.8$ \\
\hline Percent dry weight in fat & $96.5 \pm 1.58$ & $54.0 \pm 15.0$ & $44.9 \pm 25.9$ \\
\hline Liver somatic index & $3.0 \pm 0.4^{\mathrm{a}}$ & $0.86 \pm 0.98^{\mathrm{b}}$ & $2.92 \pm 1.36^{\mathrm{a}}$ \\
\hline
\end{tabular}

${ }^{1}$ Values are presented as arithmetic mean \pm standard deviation.

a,b different letters indicate site differences detected by one-way ANOVA.

the USEPA's Environmental Monitoring and Assessment Program (Wade et al., 1988, 1993; Sericano et al., 1990). These procedures were modified by adding $10 \times$ lower surrogate concentrations and injecting $10 \times$ less volume during gas chromatography to provide the lower method detection limits (MDLs) required for arctic samples. Briefly, tissues $(0.5-15 \mathrm{~g}$ wet wt.) were extracted with $\mathrm{CH}_{2} \mathrm{Cl}_{2}$, fractionated using alumina:silica open column chromatography, purified by HPLC, and analyzed using dual column fused-silica capillary column gas chromatography with an electron capture detector (GC-ECD) using a Hewlett-Packard 5880A GC in the splitless mode.

\section{Statistical Analysis}

All contaminant data were $\log _{10}$-transformed as preliminary data exploration revealed proportional relationships between means and standard deviations for some analytes. As recommended by Hebert and Keenleyside (1995), data were not normalized for lipid (or percent moisture for metals) because preliminary data analysis did not support a strong or consistent correlation between percent lipid and the most frequently detected analytes $\left(\mathrm{r}_{\mathrm{s}}<0.50\right)$. After equal variance assumption validation using the modified Levine test, site and sex differences were examined by one-way ANOVA, followed by the NeumanKeuls post-hoc multiple comparison test (software: NCSS, Kaysville, Utah). A significance level of 0.05 was applied.

Frequency of detection data was based only on data that passed the quality assurance (QA) test, and occurred above the MDL, which varied from sample to sample because of varying initial wet weights. There were numerous occasions in which an analyte was detected in a sample below the MDL. Calculations of concentration data include those samples in which the analyte was detected below the MDL, but do not include samples for which the analyte was not detected. As high variability in analyte concentrations has been observed in field studies, outliers were considered to represent variability among individuals and were therefore not eliminated.

\section{RESULTS}

Squirrels were trapped and sampled from all sites over the three-year study period. Characteristics of the sampled populations are presented in Table 1. Squirrels were collected after the breeding and parturition season, and sampled individuals were restricted to adults and subadults $(>400 \mathrm{~g}$ ). Among general health parameters, only percent lipid in fat and liver somatic index showed site-related differences.

\section{Contaminant Burdens in Liver and Fat Tissue}

Heavymetals: Concentrations of nonessential metals ranged from below detection to $32 \mu \mathrm{g} / \mathrm{g}$ wet weight, with average concentrations below $0.5 \mu \mathrm{g} / \mathrm{g}$ wet wt. (Table 2). The rank concentrations of metals was $\mathrm{Zn}>\mathrm{Cu}>\mathrm{Cd}>\mathrm{Se}>\mathrm{As}>\mathrm{Ni}$, $\mathrm{Pb}>\mathrm{Hg}$. Only a very small percentage of the mercury was present as methyl-Hg, typically less than $0.5 \%$. Site differences were observed for $\mathrm{Hg}, \mathrm{Ni}$, and $\mathrm{Pb}$, but patterns were not consistent among metals. $\mathrm{Hg}$ was higher in squirrels from Feniak Lake than Elusive Lake. Ni was higher in squirrels from Feniak and Elusive Lakes than in those from the Schrader Lake vicinity. $\mathrm{Pb}$ was higher in Feniak and Schrader squirrels than in those collected at Elusive Lake. $\mathrm{Cd}, \mathrm{Cu}$, and $\mathrm{Hg}$ concentrations increased with squirrel length (Table 3), but only $\mathrm{Cd}$ increased with weight. A negative correlation was observed between Se and weight. LSI was positively correlated with $\mathrm{Ni}$ and $\mathrm{Se}$, but negatively correlated with $\mathrm{Pb}$. There were no significant correlations between metals and percent body weight as fat. Metals tended to increase in concert with one another, as evidenced by the positive correlations between $\mathrm{As}$ and $\mathrm{Cd}, \mathrm{Cd}$ and $\mathrm{Cu}, \mathrm{Cu}$ and $\mathrm{Zn}$, and $\mathrm{Hg}$ and $\mathrm{Pb}$ (Table 3).

Organochlorines in liver: $\mathrm{HCB}$, trans-nonachlor, $\mathrm{p}, \mathrm{p}$ DDE and PCBs 138 and 170 were most frequently detected in arctic ground squirrel livers (Table 4). These analytes were present in squirrels from all three sites. Compounds that were found infrequently included $\gamma-, \alpha-$, and oxy-chlordane, endrin, mirex, 1,2,3,4- and 1,2,4,5-tetrachlorobenzene (TCB), pentachlorobenzene, p,p'-DDT, p,p'-DDD, and PCBs 8, 52, 
TABLE 2. Heavy metal concentrations in arctic ground squirrel liver $(\mu \mathrm{g} / \mathrm{g}$ wet weight).

\begin{tabular}{|c|c|c|c|c|c|c|c|}
\hline Analyte & Site & $*$ & $\mathrm{~N}$ & Mean & SD & Min. & Max. \\
\hline \multirow[t]{3}{*}{ Arsenic } & Elusive & & 13 & 0.18 & 0.27 & 0.03 & 1.02 \\
\hline & Feniak & & 13 & 0.08 & 0.08 & 0.03 & 0.30 \\
\hline & Schrader & & 23 & 0.11 & 0.10 & 0.01 & 0.35 \\
\hline \multirow[t]{3}{*}{ Cadmium } & Elusive & & 13 & 0.37 & 0.49 & 0.05 & 1.74 \\
\hline & Feniak & & 13 & 0.16 & 0.17 & 0.02 & 0.60 \\
\hline & Schrader & & 23 & 0.65 & 1.01 & 0.03 & 4.78 \\
\hline \multirow[t]{3}{*}{ Copper } & Elusive & & 13 & 5.99 & 0.68 & 4.86 & 7.50 \\
\hline & Feniak & & 13 & 5.95 & 1.07 & 4.08 & 7.85 \\
\hline & Schrader & & 23 & 6.03 & 1.30 & 4.12 & 8.35 \\
\hline \multirow{3}{*}{ Total Mercury } & Elusive & $\mathrm{a}$ & 7 & 0.02 & 0.02 & 0.01 & 0.06 \\
\hline & Feniak & $\mathrm{b}$ & 8 & 0.05 & 0.02 & 0.02 & 0.08 \\
\hline & Schrader & & 0 & & & & \\
\hline \multicolumn{3}{|c|}{$\%$ Mercury as methyl-HgElusive } & 3 & 0.37 & 0.07 & 0.30 & 0.43 \\
\hline & Feniak & & 7 & 0.13 & 0.05 & 0.06 & 0.20 \\
\hline & Schrader & & 0 & & & & \\
\hline \multirow[t]{3}{*}{ Nickel } & Elusive & $\mathrm{a}$ & 13 & 0.15 & 0.39 & 0.01 & 1.45 \\
\hline & Feniak & a & 13 & 0.09 & 0.14 & 0.03 & 0.55 \\
\hline & Schrader & $\mathrm{b}$ & 23 & 0.03 & 0.03 & 0.00 & 0.13 \\
\hline \multirow[t]{3}{*}{ Lead } & Elusive & $\mathrm{a}$ & 13 & 0.03 & 0.02 & 0.01 & 0.07 \\
\hline & Feniak & $\mathrm{b}$ & 13 & 0.18 & 0.22 & 0.01 & 0.83 \\
\hline & Schrader & $\mathrm{b}$ & 23 & 0.13 & 0.08 & 0.02 & 0.28 \\
\hline \multirow[t]{3}{*}{ Selenium } & Elusive & & 7 & 0.26 & 0.21 & 0.09 & 0.56 \\
\hline & Feniak & & 5 & 0.23 & 0.06 & 0.17 & 0.32 \\
\hline & Schrader & & 0 & & & & \\
\hline \multirow[t]{3}{*}{$\overline{Z i n c}$} & Elusive & & 13 & 32.60 & 3.83 & 26.85 & 38.20 \\
\hline & Feniak & & 13 & 33.26 & 7.85 & 22.20 & 50.09 \\
\hline & Schrader & & 23 & 35.19 & 8.61 & 24.75 & 61.38 \\
\hline
\end{tabular}

Concentration values include all samples in which analyte was detected, using analytical estimates for samples less than the method detection limit.

* different letters indicate site differences detected by one-way ANOVA.

87,153 , and 180 . The following analytes were not detected above the MDL in any squirrel liver samples: $\alpha-\mathrm{HCH}$, $\beta-\mathrm{HCH}, \delta-\mathrm{HCH}$, heptachlor, aldrin, endosulfan 2 , pentachloroanisole, and o,p'-DDE, o,p'-DDD, o,p'-DDT and PCBs 29,44 , and 200 . Greater than $85 \%$ of $\sum$ DDTs was found as p,p'-DDE in most liver samples.

Consistent differences among sites or years of sampling for major analytes were not apparent. In liver, p,p'-DDE was the only OC showing a site effect (Schrader $>$ Elusive and Feniak). PCBs were most often detected in Feniak squirrels, and the suite of PCB congeners found in squirrels from Schrader was reduced relative to the other two sites. PCBs 66, 101, 128, 153, 195, and 209 were not detected in squirrel livers from the Schrader site.

Among the most frequently detected analytes, there were no sex-related differences in OC concentrations. Although correlations between concentrations of $\mathrm{OCs}$ and squirrel weight were also not significant, negative correlations were observed between the percentage of body weight as fat and p, $\mathrm{p}^{\prime}$-DDE, trans-nonachlor, and PCBs 138 and 170 (see Table 3). PCBs 138, 170, and 180 increased in concert with one another.

Organochlorines in Fat: HCB, p,p'-DDE, and PCBs 138 and 153 were most frequently found in squirrel fat samples
TABLE 3. Significant Spearman correlation coefficients among health parameters and liver contaminant concentrations.

\begin{tabular}{|c|c|c|c|}
\hline Variable 1 & Variable 2 & Coefficient & $\mathrm{N}$ \\
\hline Weight & Total Length & 0.74 & 43 \\
\hline Weight & LSI & -0.38 & 30 \\
\hline Total Length & $\%$ body wt. as fat & 0.56 & 38 \\
\hline Total Length & $\%$ lipid in liver & 0.35 & 40 \\
\hline$\%$ body wt. as fat & $\%$ lipid in liver & 0.46 & 35 \\
\hline Weight & $\mathrm{Cd}$ & 0.55 & 42 \\
\hline Weight & $\mathrm{Se}$ & -0.68 & 9 \\
\hline Total Length & $\mathrm{Cd}$ & 0.42 & 42 \\
\hline Total Length & $\mathrm{Cu}$ & 0.33 & 42 \\
\hline Total Length & $\mathrm{Hg}$ & 0.63 & 11 \\
\hline LSI & $\mathrm{Ni}$ & 0.48 & 30 \\
\hline LSI & $\mathrm{Pb}$ & -0.48 & 30 \\
\hline LSI & $\mathrm{Se}$ & 0.80 & 9 \\
\hline LSI & t-nonachlor & -0.53 & 18 \\
\hline LSI & $\mathrm{p}, \mathrm{p}^{\prime}-\mathrm{DDE}$ & -0.50 & 21 \\
\hline LSI & РCB 170 & -0.70 & 18 \\
\hline LSI & PCB 138 & -0.64 & 25 \\
\hline$\%$ body wt. as fat & t-nonachlor & -0.53 & 26 \\
\hline$\%$ body wt. as fat & p,p'-DDE & -0.41 & 31 \\
\hline$\%$ body wt. as fat & РCB 170 & -0.58 & 18 \\
\hline$\%$ body wt. as fat & PCB 138 & -0.44 & 24 \\
\hline As & $\mathrm{Cd}$ & 0.33 & 42 \\
\hline $\mathrm{Cd}$ & $\mathrm{Cu}$ & 0.35 & 42 \\
\hline $\mathrm{Cu}$ & $\mathrm{Zn}$ & 0.46 & 42 \\
\hline $\mathrm{Hg}$ & $\mathrm{Pb}$ & 0.80 & 11 \\
\hline $\mathrm{Ni}$ & $\mathrm{p}, \mathrm{p}^{\prime}-\mathrm{DDE}$ & -0.37 & 32 \\
\hline $\mathrm{Ni}$ & РCB 180 & 0.85 & 8 \\
\hline $\mathrm{Pb}$ & $\mathrm{HCB}$ & 0.50 & 29 \\
\hline $\mathrm{Pb}$ & $\mathrm{p}, \mathrm{p}^{\prime}-\mathrm{DDE}$ & 0.35 & 33 \\
\hline $\mathrm{Zn}$ & $\mathrm{HCB}$ & 0.46 & 29 \\
\hline $\mathrm{Zn}$ & PCB 153 & -0.94 & 6 \\
\hline PCB 170 & PCB 138 & .0 .94 & 15 \\
\hline PCB 180 & PCB 138 & 0.83 & 6 \\
\hline
\end{tabular}

(Table 5). Unfortunately, fat samples from Elusive Lake squirrels rarely met the QA criteria for reporting. Between $71 \%$ and $100 \%$ of $\Sigma \mathrm{HCHs}$ was present as $\alpha-\mathrm{HCH}$ in squirrel fat samples in which $\alpha-\mathrm{HCH}$ was detected. Likewise, p,p'DDE accounted for more than 90\% of EDDTs in 6 of 13 squirrel fat samples in which this isomer was detected. The following analytes were not detected above the MDL in any fat samples: $\gamma-, \beta$-, and $\delta-\mathrm{HCH}$, heptachlor, aldrin, endosulfan 2 , pentachloroanisole, and o, p'-DDE, o, p'-DDD, and o, p'DDT. Total PCBs in fat was negatively correlated with squirrel weight $\left(\mathrm{r}_{\mathrm{s}}=-0.65, p<0.01, \mathrm{n}=18\right)$. This relationship was largely driven by the more chlorinated congeners (PCBs 153 and 180).

\section{DISCUSSION}

Our results indicate a low level of heavy metal accumulation in arctic ground squirrels, similar to that reported for other North American arctic terrestrial herbivores. In caribou, lemming, and ptarmigan livers from the Canadian High Arctic, nickel was not detectable in all species, and lead concentrations were not detectable in ptarmigan and were 1.0 and $3.5 \mu \mathrm{g} / \mathrm{g}$ wet wt. or lower in lemming and caribou respectively (Shaw and Gunn, 1981). Livers of caribou from 
TABLE 4. Organochlorine concentrations in arctic ground squirrel liver ( $\mu \mathrm{g} / \mathrm{g}$ wet weight).

\begin{tabular}{|c|c|c|c|c|c|c|c|c|c|c|c|c|c|c|c|}
\hline Analyte & Site & $*$ & $\mathrm{~N}$ & Mean & SD 1 & Minimum & Maximum & Analyte & Site & $*$ & $\mathrm{~N}$ & Mean & SD & Minimum & Maximum \\
\hline \multirow[t]{3}{*}{$\mathrm{HCB}$} & Elusive & & 11 & 0.24 & 0.22 & 0.05 & 0.82 & \multirow[t]{3}{*}{ PCB 87} & \multicolumn{2}{|l|}{ Elusive } & 5 & 0.05 & 0.02 & 0.03 & 0.07 \\
\hline & Feniak & & 9 & 0.48 & 0.31 & 0.17 & 0.95 & & \multirow{2}{*}{\multicolumn{2}{|c|}{$\begin{array}{l}\text { Feniak } \\
\text { Schrader }\end{array}$}} & 2 & 1.71 & 2.28 & 0.10 & 3.33 \\
\hline & Schrader & & 12 & 0.41 & 0.21 & 0.15 & 0.96 & & & & 2 & 3.09 & 3.81 & 0.40 & 5.79 \\
\hline$\alpha-\mathrm{HCH}$ & all & & 0 & & & & & \multirow[t]{3}{*}{ РCB 101} & \multirow{3}{*}{\multicolumn{2}{|c|}{$\begin{array}{l}\text { Elusive } \\
\text { Feniak } \\
\text { Schrader }\end{array}$}} & 3 & 0.22 & 0.16 & 0.05 & 0.38 \\
\hline \multirow[t]{3}{*}{$\gamma-\mathrm{HCH}$} & \multirow{3}{*}{\multicolumn{2}{|c|}{$\begin{array}{l}\text { Elusive } \\
\text { Feniak } \\
\text { Schrader }\end{array}$}} & 4 & 1.36 & 0.61 & 0.68 & 1.96 & & & & 2 & 0.19 & 0.01 & 0.18 & 0.20 \\
\hline & & & 0 & 1.00 & 0.01 & 0.00 & 1.70 & & & & 0 & & & & \\
\hline & & & 2 & 1.85 & 2.13 & 0.35 & 3.36 & \multirow[t]{3}{*}{ PCB 105} & \multirow{3}{*}{\multicolumn{2}{|c|}{$\begin{array}{l}\text { Elusive } \\
\text { Feniak } \\
\text { Schrader }\end{array}$}} & 3 & 0.08 & 0.09 & 0.01 & 0.18 \\
\hline \multirow[t]{3}{*}{ dieldrin } & Elusive & & 7 & 10.91 & 12.46 & 0.74 & 28.70 & & & & 2 & 0.41 & 0.16 & 0.30 & 0.53 \\
\hline & Feniak & & 7 & 1.53 & 1.68 & 0.22 & 4.90 & & & & 0 & & & & \\
\hline & Schrader & & 7 & 14.42 & 25.55 & 0.51 & 71.04 & \multirow[t]{3}{*}{ РCB 118} & \multirow{3}{*}{\multicolumn{2}{|c|}{$\begin{array}{l}\text { Elusive } \\
\text { Feniak } \\
\text { Schrader }\end{array}$}} & 3 & 0.17 & 0.16 & 0.06 & 0.35 \\
\hline endosulfan-2 & all & & 0 & & & & & & & & 2 & 1.20 & 0.88 & 0.58 & 1.83 \\
\hline \multirow{3}{*}{ heptachlor epoxide } & Elusive & & 0 & & & & & & & & 0 & & & & \\
\hline & Feniak & & 0 & & & & & \multirow[t]{3}{*}{ PCB 128} & \multirow{3}{*}{\multicolumn{2}{|c|}{$\begin{array}{l}\text { Elusive } \\
\text { Feniak } \\
\text { Schrader }\end{array}$}} & 2 & 0.10 & 0.06 & 0.06 & 0.14 \\
\hline & Schrader & & 4 & 2.80 & 1.67 & 0.54 & 4.42 & & & & 1 & 0.11 & & & \\
\hline \multirow{3}{*}{ trans-nonachlor } & Elusive & & 9 & 0.87 & 1.28 & 0.15 & 4.16 & & & & 0 & & & & \\
\hline & Feniak & & 7 & 1.42 & 0.67 & 0.78 & 2.76 & PCB 138 & Elusive & & 4 & 0.48 & 0.10 & 0.39 & 0.62 \\
\hline & Schrader & & 13 & 3.43 & 4.34 & 0.08 & 15.55 & & Feniak & & 10 & 1.45 & 0.95 & 0.29 & 2.96 \\
\hline p,p'-DDE & Elusive & $\mathrm{a}$ & 13 & 1.51 & 1.56 & 0.33 & 5.57 & & Schrader & & 16 & 3.53 & 3.84 & 0.09 & 12.25 \\
\hline & Feniak & $\mathrm{a}$ & 6 & 0.86 & 1.11 & 0.19 & 3.10 & PCB 153 & Elusive & & 4 & 0.85 & 0.17 & 0.66 & 1.02 \\
\hline & Schrader & $\mathrm{b}$ & 17 & 4.47 & 4.75 & 0.12 & 13.63 & & Feniak & & 4 & 1.96 & 2.50 & 0.21 & 5.62 \\
\hline$\overline{\sum D^{\prime} T^{1}}{ }^{1}$ & Elusive & & 13 & 6.13 & 9.17 & 0.34 & 34.08 & & Schrader & & 0 & & & & \\
\hline & Feniak & & 6 & 1.43 & 1.93 & 0.19 & 5.16 & РCВ 170 & Elusive & & 4 & 0.52 & 0.16 & 0.36 & 0.68 \\
\hline & Schrader & & 17 & 12.25 & 12.14 & 0.12 & 39.76 & & Feniak & & 6 & 1.06 & 0.89 & 0.44 & 2.83 \\
\hline$\overline{\sum \mathrm{HCHs}^{2}}$ & Elusive & & 4 & 136 & 0.61 & 0.68 & 196 & & Schrader & & 16 & 3.53 & 3.84 & 0.09 & 12.25 \\
\hline & Feniak & & 0 & & & & & РCB 180 & Elusive & & 2 & 0.34 & 0.27 & 0.15 & 0.53 \\
\hline & Schrader & & 2 & 1.85 & 2.13 & 0.35 & 3.36 & & Feniak & & 4 & 1.27 & 1.53 & 0.25 & 3.54 \\
\hline$\overline{\sum \text { chlordanes }^{3}}$ & Elusive & & 2 & 025 & 008 & 0.19 & 030 & & Schrader & & 2 & 0.13 & 0.07 & 0.08 & 0.18 \\
\hline & Feniak & & 1 & $\begin{array}{l}0.25 \\
1.12\end{array}$ & 0.00 & 0.17 & 0.00 & PCB 187 & Elusive & & 3 & 0.19 & 0.03 & 0.17 & 0.23 \\
\hline & Schrader & & 9 & 4.28 & 6.38 & 0.08 & 19.96 & & Feniak & & 2 & 0.17 & 0.03 & 0.15 & 0.19 \\
\hline PCB 8 & Elusive & & 1 & 3.57 & & 3.57 & 3.57 & & Schrader & & 1 & 0.14 & & 0.14 & 0.14 \\
\hline & Feniak & & 4 & 18.19 & 21.48 & 0.51 & 48.69 & PCB 195 & Elusive & & 1 & 0.16 & & & \\
\hline & Schrader & & 2 & 0.48 & 0.01 & 0.47 & 0.49 & & Feniak & & 1 & 0.11 & & & \\
\hline PCB 18 & Elusive & & 0 & & & & & & Schrader & & 0 & & & & \\
\hline & Feniak & & 1 & 0.19 & & 0.19 & 0.19 & $\underline{\text { PCB } 200}$ & all & & 0 & & & & \\
\hline & Schrader & & 2 & 1.16 & 0.94 & 0.49 & 1.82 & PCB 206 & Elusive & & 1 & 0.05 & & 0.05 & 0.05 \\
\hline PCB 28 & Elusive & & 2 & 0.28 & 0.15 & 0.17 & 0.39 & & Feniak & & 0 & & & & \\
\hline & Feniak & & 0 & & & & & & Schrader & & 0 & & & & \\
\hline & Schrader & & 3 & 0.56 & 0.65 & 0.07 & 1.30 & РCB 209 & Elusive & & 1 & 0.11 & & 0.11 & 0.11 \\
\hline PCB 29 & all & & 0 & & & & & & Feniak & & 1 & 0.09 & & 0.09 & 0.09 \\
\hline PCB 44 & all & & 0 & & & & & & Schrader & & 0 & & & & \\
\hline $\begin{array}{l}\text { PCB } 52 \\
\end{array}$ & Elusive & & 3 & & & & & $\sum \mathrm{PCBs}^{4}$ & Elusive & & 1 & 2.09 & & 2.09 & 2.09 \\
\hline & Feniak & & 6 & 0.65 & 0.39 & 0.27 & $\begin{array}{l}1.14 \\
1.35\end{array}$ & & Feniak & & 11 & 9.72 & 14.31 & 1.47 & 50.04 \\
\hline & Schrader & & 3 & 0.20 & 0.01 & 0.20 & 0.21 & & Schrader & & 10 & 5.72 & 4.70 & 0.74 & 12.74 \\
\hline PCB 66 & Elusive & & 2 & 1.56 & 0.56 & 1.16 & 1.95 & & & & & & & & \\
\hline & Feniak & & 1 & 0.49 & & 0.49 & 0.49 & & & & & & & & \\
\hline & Schrader & & 0 & & & & & & & & & & & & \\
\hline
\end{tabular}

Concentration values include all samples in which analyte was detected, using analytical estimates for samples less than the method detection limit.

* different letters indicate site differences detected by one-way ANOVA.

${ }^{1} \sum$ DDTs $=$ p,p'-DDE, p,p'-DDD, p,p'-DDT.

${ }^{2} \sum \mathrm{HCHs}=\alpha \mathrm{HCH}+\gamma \mathrm{HCH}$.

${ }^{3} \sum$ chlordanes $=\alpha$ chlordane $+\gamma$ chlordane + trans -nonachlor + heptachlor epoxide.

${ }^{4} \sum \mathrm{PCBs}=$ sum of congeners: 8, 18, 28, 29, 44, 52, 66, 87, 101, 105, 118, 128, 138, 153, 170, 187, 195, $200,206,209$.

the eastern Canadian Arctic had similar concentrations of $\mathrm{Ni}$, $\mathrm{Pb}$, and $\mathrm{Hg}$, but $\mathrm{Cd}$ concentrations five times higher than those we observed in ground squirrels (Elkin and Bethke, 1995). We suspect that the higher level of $\mathrm{Cd}$ in caribou reflects differences in age and concentrations of liver metalbinding proteins (metallothioneins) between caribou and squirrels. In polar bears, $\mathrm{Cd}$ and $\mathrm{Hg}$ concentrations in livers from Svalbard were also within the range observed in ground squirrels (Norheim et al., 1992), while four bears from the Canadian High Arctic had $\mathrm{Hg}$ concentrations in liver in excess of $100 \mu \mathrm{g} / \mathrm{g}$ wet wt. (Norstrom et al., 1986). Although $\mathrm{Hg}$ and other heavy metals can be atmospherically transported, 
TABLE 5. Organochlorine concentrations in arctic ground squirrel fat ( $\mu \mathrm{g} / \mathrm{g}$ wet weight).

\begin{tabular}{|c|c|c|c|c|c|c|c|c|c|c|c|c|c|}
\hline Analyte & Site & $\mathrm{N}$ & Mean & SD & Minimum & Maximum & Analyte & Site & $\mathrm{N}$ & Mean & SD & Minimum & Maximum \\
\hline \multirow[t]{3}{*}{$\mathrm{HCB}$} & Elusive & 0 & & & & & PCB 101 & all & 18 & n.d. & & & \\
\hline & Feniak & 8 & 2.0 & 2.1 & $<\mathrm{mdl}$ & 5.1 & \multirow[t]{3}{*}{ PCB 105} & Elusive & 1 & 0.2 & \multirow{3}{*}{2.7} & \multirow{3}{*}{0.5} & \multirow{3}{*}{4.3} \\
\hline & Schrader & 5 & 6.5 & 2.5 & 4.6 & 10.9 & & Feniak & 2 & 2.4 & & & \\
\hline \multirow[t]{3}{*}{$\alpha \mathrm{HCH}$} & Elusive & 0 & & & & & & Schrader & 5 & n.d. & & & \\
\hline & Feniak & 9 & 1.0 & 1.1 & $<\mathrm{mdl}$ & 3.0 & \multirow{3}{*}{ PCB 118} & Elusive & 1 & 0.5 & \multirow{3}{*}{6.9} & \multirow{3}{*}{0.7} & \multirow{3}{*}{12.9} \\
\hline & Schrader & 5 & 1.6 & 1.0 & $<$ mdl & 2.8 & & Feniak & 3 & 5.0 & & & \\
\hline$\gamma \mathrm{HCH}$ & all & 13 & n.d. & & & & & Schrader & 0 & n.d. & & & \\
\hline \multirow[t]{3}{*}{ dieldrin } & Elusive & 0 & & & & & \multirow[t]{3}{*}{ PCB 128} & Elusive & 1 & 0.2 & \multirow{3}{*}{0.4} & \multirow{3}{*}{1.1} & \multirow{3}{*}{1.6} \\
\hline & Feniak & 8 & 0.0 & 0.1 & $<\mathrm{mdl}$ & 0.3 & & Feniak & 2 & 1.3 & & & \\
\hline & Schrader & 5 & 0.5 & 0.8 & $<\mathrm{mdl}$ & 1.8 & & Schrader & 5 & n.d. & & & \\
\hline \multirow[t]{3}{*}{ endosulfan-2 } & Elusive & 2 & 0.1 & 0.2 & $<\mathrm{mdl}$ & 0.3 & \multirow[t]{3}{*}{ PCB 138} & Elusive & 2 & 0.4 & 0.1 & 0.4 & 0.5 \\
\hline & Feniak & 10 & n.d. & & & & & Feniak & 10 & 4.2 & 7.5 & 0.0 & 18.4 \\
\hline & Schrader & 5 & n.d. & & & & & Schrader & 5 & 0.5 & 0.4 & 0.3 & 1.1 \\
\hline \multirow[t]{3}{*}{ heptachlor epoxide } & Elusive & 0 & & & & & \multirow[t]{3}{*}{ PCB 153} & Elusive & 2 & 0.5 & 0.1 & 0.4 & 0.6 \\
\hline & Feniak & 8 & 0.3 & 0.2 & $<\mathrm{mdl}$ & 0.5 & & Feniak & 10 & 13.6 & 24.3 & 0.4 & 62.6 \\
\hline & Schrader & 5 & 0.6 & 0.5 & $<\mathrm{mdl}$ & 1.3 & & Schrader & 2 & 1.3 & 0.1 & 1.3 & 1.4 \\
\hline \multirow{3}{*}{ trans-nonachlor } & Elusive & 2 & 0.1 & 0.2 & $<\mathrm{mdl}$ & 0.3 & \multirow{3}{*}{ PCB 170} & Elusive & 1 & 0.2 & \multirow{3}{*}{7.3} & \multirow{3}{*}{1.7} & \\
\hline & Feniak & 11 & 0.5 & 0.5 & $<\mathrm{mdl}$ & 1.3 & & Feniak & 2 & 6.8 & & & 12.0 \\
\hline & Schrader & 5 & 0.6 & 0.5 & $<$ mdl & 1.4 & & Schrader & 0 & n.d. & & & \\
\hline p,p'-DDE & Elusive & 0 & & & & & PCB 180 & Elusive & 2 & 0.3 & 0.3 & 0.2 & 0.5 \\
\hline & Feniak & 8 & 3.2 & 3.7 & $<\mathrm{mdl}$ & 9.4 & & Feniak & 11 & 9.0 & 9.4 & 15.5 & 0.2 \\
\hline & Schrader & 5 & 7.9 & 3.9 & 3.3 & 13.5 & & Schrader & 4 & 0.6 & 0.3 & 0.2 & 0.9 \\
\hline$\overline{\sum \text { DDTs }^{1}}$ & Elusive & 0 & & & & & $\overline{\mathrm{PCB}} 187$ & Elusive & 1 & 0.1 & & & \\
\hline & Feniak & 1 & 5.3 & & & & & Feniak & 2 & 0.4 & 0.0 & 0.3 & 0.4 \\
\hline & Schrader & 0 & & & & & & Schrader & 1 & 0.1 & & & \\
\hline$\overline{\sum \mathrm{HCHs}^{2}}$ & Elusive & 0 & & & & & PCB 195 & Elusive & 0 & & & & \\
\hline & Feniak & 8 & 1.1 & 1.1 & $<\mathrm{mdl}$ & 3.0 & & Feniak & 1 & 1.9 & & & \\
\hline & Schrader & 5 & 1.6 & 1.0 & $<\mathrm{mdl}$ & 2.8 & & Schrader & 5 & n.d. & & & \\
\hline PCB 8 & all & 14 & n.d. & & & & PCB 200 & all & 18 & n.d. & & & \\
\hline РCB 18 & all & 18 & n.d. & & & & $\overline{\mathrm{PCB}} 206$ & Elusive & 1 & 0.1 & & & \\
\hline PCB 28 & all & 18 & n.d. & & & & & Feniak & 4 & 1.1 & 1.4 & 0.1 & 3.0 \\
\hline PCB 29 & all & 18 & n.d. & & & & & Schrader & 5 & n.d. & & & \\
\hline PCB 44 & all & 18 & n.d. & & & & PCB 209 & Elusive & 0 & n.d. & & & \\
\hline PCB 52 & all & 18 & n.d. & & & & $1 C D 20$ & Feniak & 2 & 0.4 & 0.2 & 0.3 & 0.5 \\
\hline PCB 66 & all & 13 & n.d. & & & & & Schrader & 5 & n.d. & & & \\
\hline PCB 87 & Elusive & 1 & 0.1 & & & & & & & & & & \\
\hline & Feniak & 0 & n.d. & & & & & & & & & & \\
\hline & Schrader & 0 & n.d. & & & & & & & & & & \\
\hline
\end{tabular}

Concentration values include all samples in which analyte was detected, using analytical estimates for samples less than the method detection limit.

n.d. = not detected.

$<$ mdl $=$ less than method detection limit.

${ }^{1} \sum$ DDTs $=$ p,p'-DDE, p,p'-DDD, p,p'-DDT.

${ }^{2} \sum \mathrm{HCHs}=\alpha \mathrm{HCH}+\gamma \mathrm{HCH}$.

deposition zones are usually not extensive (Stickel, 1973; Landers et al., in press). Local and regional areas of elevated heavy metal contamination in arctic fauna are more likely related to mineral-rich geologic areas (Norstrom et al., 1986; Allen-Gil et al., 1997).

The occurrence of OCs in a herbivorous rodent from remote areas of northern Alaska highlights the spatial and temporal pervasiveness of OC contamination. This finding supports the global distillation model developed by Wania and Mackay (1993, 1995, 1996), and empirical data for terrestrial (Simonich and Hites, 1995) and freshwater ecosystems (Muir et al., 1995; Allen-Gil et al., 1997). While the magnitude of contamination in arctic ground squirrels is not high relative to other areas and species, it represents an important source for food chain transfer and potential biomagnification (Larsson et al., 1990a).

Concentrations of OCs in ground squirrels from northern Alaska are not elevated relative to those in herbivorous small mammals from other areas or in other arctic wildlife species. In a review of arctic terrestrial contamination, Thomas et al. (1992) reported concentrations of OCs in caribou, hare, ptarmigan, and musk-ox from the Canadian Arctic. For $\Sigma$ DDTs and $\Sigma$ PCBs, concentrations in arctic herbivores throughout North America are typically below $50 \mathrm{ng} / \mathrm{g}$ wet wt. and $100 \mathrm{ng} / \mathrm{g}$ wet wt. respectively. Concentrations of OC pesticide in arctic ground squirrels were in the same range as those reported for carcass samples from rock ptarmigan (Lagopus mutus), a common herbivorous arctic ground bird 
$(<10 \mathrm{ng} / \mathrm{g}$ wet wt. for individual analytes, according to Snyder-Conn and Lubinski, 1993). Maximum concentrations of persistent organic contaminants in arctic ground squirrel fat were lower than approximate mean values in Canadian caribou fat $(\mathrm{HCB}=26 \mathrm{ng} / \mathrm{g}$ wet wt., $\Sigma \mathrm{HCH}=20, \Sigma \mathrm{PCBs}=$ 16, according to Elkin and Bethe, 1995). Reindeer liver from northern Sweden were also found to contain less than $1 \mathrm{ng} / \mathrm{g}$ wet wt. of most OCs, with the exception of PCB 153, which occurred at concentrations of $20 \mathrm{ng} / \mathrm{g}$ in some samples (Villeneuve et al., 1985).

In addition to predicted species differences in OC accumulation based on food web position and percent body weight as fat, geographic differences have also been observed. In polar bears, OC tissue concentrations decreased with increasing latitude and from east to west (Norstrom et al., 1988). The same pattern was observed in sediment cores for mercury, an atmospherically transported heavy metal (Lockhart et al., 1995).

Maximum $\sum$ DDTs in arctic ground squirrel fat and liver were $40 \mathrm{ng} / \mathrm{g}$ wet wt., compared to mean total body residues of $\sum$ DDTs in mice and voles from reference sites in Maine of $30 \mathrm{ng} / \mathrm{g}$ wet wt. (Dimond and Sherburne, 1969). Maximum $\Sigma$ PCBs in arctic ground squirrel livers were $50 \mathrm{ng} / \mathrm{g}$ wet wt., compared to $1300 \mathrm{ng} / \mathrm{g}$ wet wt. in small mammal livers from an uncontaminated site in South Dakota (Greichus and Dohman, 1980).

Although the number of analyzed samples is too low to make definitive statements, it appears that $\mathrm{OC}$ pesticide and PCB residues in arctic herbivores are typically one to two orders of magnitude lower than values reported for arctic predators and marine mammals. For example, maximum $\sum$ PCBs in arctic ground squirrel liver was $4.3 \mu \mathrm{g} / \mathrm{g}$ lipid, compared to $12.5 \mu \mathrm{g} / \mathrm{g}$ lipid in the livers of Svalbard fox (Norheim, 1978). Ringed seals, beluga whales, and polar bears sampled in Arctic regions frequently have contaminant burdens that are a hundred times higher than those observed in arctic ground squirrels (Bowes and Jonkel, 1975; Muir et al., 1988; Norstrom et al., 1988; Oehme et al., 1988; Norheim et al., 1992; Thomas et al., 1992; Cameron and Weis, 1993).

The negative correlations between tissue OC concentrations and body fat that we observed in squirrels likely reflect several processes. First, as the mass of body fat increases, a larger volume of tissue is available for OC deposition (Leatherland et al., 1979; Kawai et al., 1988). Second, shifting dietary habits may result in concurrent, but unassociated changes in OC uptake and eliminations, as well as body fat content. A third possibility is that OC burdens altered lipid metabolism in squirrels. Laboratory studies with fish have shown a decrease in carcass lipid following PCB exposure (Leatherland and Sonstegard, 1979; Leatherland et al., 1979).

A fourth possibility is that these results are an artifact of correlation analysis on multivariate data sets, for which distinguishing between ecologically meaningful and spurious relationships is not always possible.

The lack of sex-related differences in OC concentrations in ground squirrels differs from results for fish and marine mammal species (Norheim et al., 1992; Sjim et al., 1992;
Larsson et al., 1993). This difference is probably explained by the more similar pattern of fat deposition and utilization between the sexes in ground squirrels relative to nonhibernating species. In squirrels, both males and females have dramatic annual fluctuations in fat reserves: males increase their percent body weight as fat from $11 \%$ to $44 \%$ and females from $25 \%$ to $61 \%$ over the course of the summer (Batzli and Sobaski, 1980). Weight loss during hibernation is slightly higher for females than for males $(8-22 \%$ versus 25 $32 \%$ ), presumably because females tend to hibernate longer than males (McLean and Towns, 1981).

\section{CONCLUSION}

This study documents the occurrence of heavy metals and anthropogenically derived OC contaminants in remote regions of the U.S. Arctic. Heavy metal accumulation in arctic ground squirrels from Alaska is not elevated relative to other species and arctic locations, and probably reflects the minerally rich geology of northern Alaska. The fact that detectable levels of HCB, HCHs, chlordanes, DDTs and PCBs were found in a small terrestrial herbivore with a limited home range size from several sites illustrates the pervasiveness of these compounds in the global environment. Although contamination in ground squirrels is not high relative to small herbivorous mammals in the contiguous U.S. or other wildlife species in the Arctic, there may be some cause for concern and further study regarding the possibility of sublethal toxicity and biomagnification.

\section{ACKNOWLEDGEMENTS}

This research was funded by the U.S. Environmental Protection Agency through cooperative agreements with Oregon State University (CR 818187), Battelle Pacific Northwest (CR 820871) and the Geochemical and Environmental Research Group at Texas A\&M University (CR 820767). It has been subjected to the Agency's peer and administrative review and approved for publication. This manuscript is Oregon State University, Agricultural Experiment Station Technical Paper No. 11065. Mention of trade names of commercial products does not constitute endorsement or recommendation for use.

\section{REFERENCES}

ALLEN-GIL, S.M., GUBALA, C.P., LANDERS, D.H., LASORSA, B.K., CRECELIUS, E.A., and CURTIS, L.R. 1997. Heavy metal accumulation in sediment and freshwater fish in U.S. arctic lakes. Environmental Toxicology and Chemistry 16:733-741. ALLEN-GIL, S.M., GUBALA, C.P., WILSON, R., LANDERS, D.H., WADE, T.L., SERICANO, J.L., and CURTIS, L.R. In press. Organochlorine pesticides and polychlorinated biphenyls (PCBs) in sediments and biota from four U.S. Arctic lakes. Archives of Environmental Contamination and Toxicology. 
ANDERSSON, O., LINDER, C., REUTERGARDH, L., UVEMO, U., and WIDEQVIST, U. 1988. Spatial differences and temporal trends of organochlorine compounds in biota from the Northern Hemisphere. Archives of Environmental Contamination and Toxicology 17:755-765.

BABCOCK, C.A. 1986. Vegetation patterns and microtine rodent use of tundra habitats in northeastern Alaska. M.S. Thesis, University of Alaska, Fairbanks.

BARNES, B.M. 1989. Freeze avoidance in a mammal: Body temperatures below $0^{\circ} \mathrm{C}$ in an arctic hibernator. Science 244:1593-1596.

BATZLI, G.O. 1981. Populations and energetics of small mammals in the tundra ecosystem. In: Bliss, L.C., Heal, O.W., and Moore, J.J., eds. Tundra ecosystems: A comparative analysis. New York: Cambridge University Press. 377-396.

BATZLI, G.O., and SOBASKI, S.T. 1980. Distribution, abundance, weight, and foraging patterns of ground squirrels near Atkasook, Alaska. Arctic and Alpine Research 12:501-510.

BAUST, J.G., and BROWN, R.T. 1980. Heterothermy and cold acclimation in the arctic ground squirrel, Citellus undulatus. Comparative Biochemistry and Physiology 67A:447-452.

BEHIRSCH, H.W. 1984. Seasonal activity of the glycolytic enzymes in a hibernator, the arctic ground squirrel "Spermophilus parryi." Polar Biology 3:55-61.

BLIGH, E.G., and DYER, W.J. 1959. A rapid method of total lipid extraction and purification. Canadian Journal of Biochemistry and Physiology 37:911-917.

BOURGOIN, B.P., and RISK, M.J. 1987. Historical changes in lead in the Eastern Canadian Arctic, determined from fossil and modern Mya truncata shells. The Science of the Total Environment 67:287-291.

BOWES, G.W., and JONKEL, C.J. 1975. Presence and distribution of polychlorinated biphenyls (PCB) in arctic and subarctic marine food chains. Journal of the Fisheries Resource Board of Canada 32:2111-2123.

CAMERON, M., and WEIS, I.M. 1993. Organochlorine contaminants in the country food diet of the Belcher Island Inuit, Northwest Territories, Canada. Arctic 46:42-48.

CARL, E.A. 1971. Population control in arctic ground squirrels. Ecology 52:395-413.

CHAPPELL, M.A. 1981. Standard operative temperatures and cost of thermoregulation in the arctic ground squirrel, Spermophilus undulatus. Oecologia 49:397-403.

DIMOND, J.B., and SHERBURNE, J.A. 1969. Persistence of DDT in wild populations of small mammals. The American Naturalist 221:486-487.

ELKIN, B.T., and BETHKE, R.W. 1995. Environmental contaminants in caribou in the Northwest Territories, Canada. The Science of the Total Environment 160/161:307-321.

FORD, J., and BEDFORD, B.L. 1987. The hydrology of Alaskan wetlands, USA: A review. Arctic and Alpine Research 19: 209-229.

FORD, J., LANDERS, D., KUGLER, D., LASORSA, B., ALLENGIL, S., CRECELIUS, E., and MARTINSON, J. 1995. Inorganic contaminants in Arctic Alaskan ecosystems: Long-range atmospheric transport or local point sources? The Science of the Total Environment 160/161:323-335.
GEYER, H.J., SCHEUNTERT, I., RAPP, K., KETTRUP, A., KORTE, F., GREIM, H., and ROZMAN, K. 1990. Correlation between acute toxicity of 2,3,7,8-tetrachlorodibenzo- $p$-dioxin and total body fat content in mammals. Toxicology 65:97-107.

GREICHUS, Y.A., and DOHMAN, B.A. 1980. Polychlorinated biphenyl contamination in areas surrounding two transformer salvage companies, Colman, South Dakota - September 1977. Pesticide Monitoring Journal 14:26-30.

GUBALA, C.P., LANDERS, D.H., MONETTI, M., HEIT, M., WADE, T., LASORSA, B., and ALLEN-GIL, S. 1995. The rates of accumulation and chronologies of atmospherically derived pollutants in Arctic Alaska, USA. The Science of the Total Environment 160/161:347-361.

HEBERT, C.E., and KEENLEYSIDE, K.A. 1995. To normalize or not to normalize? Fat is the question. Environmental Toxicology and Chemistry 14:801-807.

HOFFMANN, R.S. 1974. Terrestrial vertebrates. In: Ives, J.D., and Barry, R.G., eds. Arctic and alpine environments. London: Methuen \& Co. 471-568.

JONES, D.M., and THEBERGE, J.B. 1983. Variation in red fox, Vulpes vulpes, summer diets in Northwest British Columbia and Southwest Yukon. Canadian Field-Naturalist 97:311-314.

KAWAI, S., FUKUSHIMA, M., MIYAZAKI, N., and TATSUKAWA, R. 1988. Relationship between lipid composition and organochlorine levels in the tissues of striped dolphin. Marine Pollution Bulletin 19:129-133.

KENAGY, G.J., and BARNES, B.M. 1988. Seasonal reproductive patterns in four coexisting rodent species from the Cascade Mountains, Washington. Journal of Mammalogy 69:274-292.

KIDD, K.A., SCHINDLER, D.W., HESSLEIN, R.H., and MUIR, D.C.G. 1995. Correlation between stable nitrogen isotope ratios and concentrations of organochlorines in biota from a freshwater food web. The Science of the Total Environment 160/161: $381-390$.

KIELL, D.J., and MILLAR, J.S. 1980. Reproduction and nutrient reserves of arctic ground squirrels. Canadian Journal of Zoology 58:416-421.

LANDERS, D.H., FORD, J., GUBALA, C.P., ALLEN-GIL, S.M., CURTIS, L., URQUHART, S.N., and OMERNIK, J.M. 1992. Arctic Contaminants Research Plan. EPA/600/R-92/210. Corvallis, Oregon: U.S. Environmental Protection Agency. 158 p.

LANDERS, D.H., FORD, J., GUBALA, C., MONETTI, M., LASORSA, B.K., and MARTINSON, J. 1995. Mercury in vegetation and lake sediments from the U.S. Arctic. Water, Air, and Soil Pollution 80:591-601.

LANDERS, D., GUBALA, C., VERTA, M., LUCOTTE, M., JOHANSSON, K., VLASOVA, T., and LOCKHART, W. In press. Using lake sediment mercury flux ratios to evaluate the regional and continental dimensions of mercury deposition in arctic and boreal systems. Atmospheric Environment.

LARSSON, P., OKLA, L., and WOIN, P. 1990a. Atmospheric transport of persistent pollutants governs uptake by holarctic terrestrial biota. Environmental Science \& Technology 24: $1599-1601$.

LARSSON, P., WOIN, P., and KNULST, J. 1990b. Differences in uptake of persistent pollutants for predators feeding in aquatic and terrestrial habitats. Holarctic Ecology 13:149-155. 
LARSSON, P., OKLA, L., and COLLVIN, L. 1993. Reproductive status and lipid content as factors in PCB, DDT and $\mathrm{HCH}$ contamination of a population of pike (Esox lucius). Environmental Toxicology and Chemistry 12:855-861.

LEATHERLAND, J.F., and SONSTEGARD, R.A. 1979. Effect of dietary Mirex and PCB (Aroclor 1254) on thyroid activity and lipid reserves in rainbow trout Salmo gairdneri Richardson. Journal of Fish Diseases 2:43-48.

LEATHERLAND, J.F., SONSTEGARD, R.A., and HOLDRIENT, M.V. 1979. Effect of dietary Mirex and PCBs on hepatosomatic index, liver lipid, carcass lipid and PCB and Mirex bioaccumulation in yearling coho salmon, Oncorhynchus kisutch. Comparative Biochemistry and Physiology 63C:243-246.

LOCKHART, W.L., WILKINSON, P., BILLECK, B.N., HUNT, R.V., WAGEMANN, R., and BRUNSKILL, G.J. 1995. Current and historical inputs of mercury to high-latitude lakes in Canada and to Hudson Bay. Water, Air, and Soil Pollution 80:603-610.

McLEAN, I.G., and TOWNS, A.J. 1981. Differences in weight changes and the annual cycle of male and female arctic ground squirrels. Arctic 34:249-254.

MUIR, D.C.G., NORSTROM, R.J., and SIMON, M. 1988. Organochlorine contaminants in arctic marine food chains: Accumulation of specific polychlorinated biphenyls and chlordane-related compounds. Environmental Science \& Technology 22:1071-1079.

MUIR, D.C.G., GRIFT, N.P., LOCKHART, W.L., WILKINSON, P., BILLECK, B.N., and BRUNSKILL, G.J. 1995. Spatial trends and historical profiles of organochlorine pesticides in arctic lake sediments. The Science of the Total Environment 160/161:447-457.

NORHEIM, G. 1978. The composition and distribution of PCB in arctic fox (Alopex lagopus) caught near Longyearbyen on Svalbard. Acta Pharmacology et Toxicology 42:7-13.

NORHEIM, G., SKAARE, J.U., and WIIG, O. 1992. Some heavy metals, essential elements, and chlorinated hydrocarbons in polar bear (Ursus maritimus) at Svalbard. Environmental Pollution 77:51-57.

NORSTROM, R.J., SCHWEISENBERG, R.E., and COLLINS, B.T. 1986. Heavy metals and essential elements in livers of the polar bear (Ursus maritimus) in the Canadian Arctic. The Science of the Total Environment 48:195-212.

NORSTROM, R.J., SIMON, M., MUIR, D.C.G., and SCHWEINSBURG, R.E. 1988. Organochlorine contaminants in arctic marine food chains: Identification, geographical distribution, and temporal trends in polar bears. Environmental Science \& Technology 22:1083-1091.

OEHME, M., FÜRST, P., KRÜGER, C., MEEMKEN, H.A., and GROEBEL, W. 1988. Presence of polychlorinated dibenzo-pdioxins, dibenzofurans, and pesticides in arctic seal from Spitzbergen. Chemosphere 17:1291-1300.

POOLE, K.G., and BOAG, D.A. 1988. Ecology of gyrfalcons, Falco rusticolus, in the central Canadian Arctic: Diet and feeding behavior. Canadian Journal of Zoology 66:334-344.

REMMERT, H. 1980. Arctic animal ecology. New York: SpringerVerlag.
SERICANO, J.L., WADE, T.L., ATLAS, E.L., and BROOKS, J.M. 1990. Historical perspective on the environmental bioavailability of DDT and its derivatives to Gulf of Mexico oysters. Environmental Science \& Technology 24:1541-1548.

SHAW, G., and GUNN, A. 1981. Element concentrations in High Arctic vegetation and some caribou and lemmings. Unpubl. report. Available from Canadian Wildlife Service, Prairie and Northern Region, 2nd floor, 4999-98th Ave., Edmonton, Alberta T6B 2X3, Canada.

SHORE, R.F., and DOUBEN, P.E.T. 1994. Predicting ecotoxicological impacts of environmental contaminants on terrestrial small mammals. Reviews of Environmental Contamination and Toxicology 134:49-89.

SIMONICH, S.L., and HITES, R.A. 1995. Global distribution of persistent organochlorine compounds. Science 269:1851-1854.

SJIM, D.T.H.M., SEINEN, W., and OPPERHUIZEN, A. 1992. Life-cycle biomagnification study in fish. Environmental Science \& Technology 26:2162-2174.

SLATTER, D.H. 1985. Textbook of small animal surgery. Philadelphia, Pennsylvania: W.B. Saunders Company.

SNYDER-CONN, E., and LUBINSKI, M. 1993. Contaminant and water quality baseline data for the Arctic National Wildlife Refuge, Alaska, 1988-1989. Vol. 2, Raw Data. Fish and Wildlife Service Technical Report, U.S. Department of Interior. NAESTR-93-03. 305 p.

STICKEL, L.F. 1973. Pesticide residues in birds and mammals. In: Edwards, C.A., ed. Environmental pollution by pesticides. New York: Plenum Press. 254-312.

STONEHOUSE, B. 1989. Polar ecology. New York: Chapman and Hall.

TALMAGE, S.S., and WALTON, B.T. 1991. Small mammals as monitors of environmental contaminants. Reviews of Environmental Contamination and Toxicology 119:47-145.

THOMAS, D.J., TRACEY, B., MARSHALL, H., and NORSTROM, R.J. 1992. Arctic terrestrial ecosystem contamination. The Science of the Total Environment 122:135-164.

VILLENEUVE, J.P., HOLM, E., and CATTINI, C. 1985. Transfer of chlorinated hydrocarbons in the food chain lichen-reindeerman. Chemosphere 14:1651-1658.

WADE, T.L., ATLAS, E.L., BROOKS, J.M., KENNICUTT, M.C., II, FOX, R.G., SERICANO, J., GARCIA-ROMERO, B., and DEFREITAS, D. 1988. NOAA Gulf of Mexico Status and Trends Program: Trace organic contaminant distribution in sediments and oysters. Estuaries 11:171-179.

WADE, T.L., BROOKS, J.M., KENNICUTT, M.C., II, McDONALD, T.J., SERICANO, J.L., and JACKSON, T.J. 1993. GERG trace organics contaminant analytical techniques. National Status and Trends program for marine environmental quality, NOAA Technical Memo NOS ORCA 71, Sampling and analytical methods of the National Status and Trends Program National Benthic Surveillance and Mussel Watch Projects 1984-1992, Vol. IV Comprehensive Descriptions of Trace Organic Analytical Methods, Silver Spring, Maryland, July 1993. 121-129.

WALSBERG, G.E. 1988. Evaluation of a nondestructive method for determining fat stores in small birds and mammals. Physiological Zoology 61:153-159. 
WANIA, F., and MACKAY, D. 1993. Global fractionation and cold condensation of low volatility organochlorine compounds in polar regions. Ambio 22:10-18.

. 1995. A global distribution model for persistent organochlorine chemicals. The Science of the Total Environment 160/161:211-232.
1996. Tracking the distribution of persistent organic pollutants. Environmental Science \& Technology 30:390A396A.

WILSON, R., ALLEN-GIL, S., GRIFFIN, D., and LANDERS, D. 1995. Organochlorine contaminants in fish from an Arctic lake in Alaska, USA. The Science of the Total Environment 160/ 161:511-519. 